\title{
INSURANCE AS A CYBERNETIC INSTITUTION \\ OF \\ SELF-REGULATION OF THE NATIONAL ECONOMY
}

\author{
Dr. Antoni Banasinski
}

The present referendum is only indirectly connected with the themes of the fifth Colloquium of ASTIN. It is more or less a memorandum about the actual work of Polish actuaries in the sector of the Insurance Theory and trying out a new conception and solution of social and economic problems of Property and Capital Insurances.

The principles and hypotheses of the cybernetic insurance theory posed in this referendum are a result of social situations in a planned economy. However, it is assumed that the cybernetic analysis of the economic rôle of insurance, and the methods of calculation resulting from this analysis, can also be applied to other social and economic situations.

In the judgment of the author, the problem under consideration would be of interest to the members of ASTIN and could possibly be included in the syllabus of one of the subsequent colloquia of ASTIN.

\section{The cybernetic insurance theory}

As is well known, there are many insurance theories and definitions that govern the meaning and significance of the specific form of social-economic activity. The scientific insurance theories were developed throughout an insurance practice already in existence for hundreds of years and from the ideas and principles worked out during this period.

Consequently, the scientific theory did govern and will continue to govern future developments of insurance and also basic calculation, adapted in insurance principles.

Nearly all definitions hypothesize that the Property (Capital) Insurance are based on the covering of losses (or on future capital 
demand) resulting from accidental circumstances. This theory does not create reserves. Furthermore, as a general characteristic of insurance, it is assumed, that the claims are to be borne by many units which are threatened by these circumstances. The insurance fund, therefore, is created by means of premiums paid by the individual insured. The level of these premiums is dependent on the insured amount (indirectly thus from the insurance value) and the extent of the risk, i.e. the degree of danger liable to the insured object.

In the same way socialist insurance theories retain the characteristics as previously mentioned although they stipulate fundamentally that the nature and importance of insurance in the sphere of national economy differ from those of the capitalist economy.

From the second mentioned characteristic of insurance, it appears that insurance has only an economic significance in cases where there exists a risk-community i.e. several separate economic units, the property of which is threatened by the same or a similar danger. Consequently, it is to be expected for instance that in the realm of socialist economy insurances are doomed to disappear, for the simple reason that-in accordance with the theory - the national capital cannot be a concrete object of insurance.

Deviating from this we have the situation that the economic function of insurance is valued on account of its close connection with the development process of National Economy. In all social situations the material production means both the national and individual goods are being threatened by unfavourable occurrences. Such occurrences lead to interruptions in social-economic processing, to weakening the communal production-power, and to the impoverishment of the people. This means that, if the development process of the National Economy has to attain the pursued end in its directrix, there has to be a regulating institution which would be able to eliminate disturbing occurrences or to compensate (equalize) unfavourable deviations of the effects caused by these occurrences. There is no doubt that one of the regulators (adjusters) of the planned course of social-economic processing, is insurance.

From the foregoing formulated cybernetic insurance theory result many practical conclusions with regard to perspectives on 
the directrix of insurance development as also to new mathematicalstatistical methods which can be applied in insurance calculations: first: the insurance funds destined to cover losses must not be built up from the contributions of economical units, the capital of which is threatened by unfavourable occurrences; the contributions could for instance be covered wholly or partially by universal social (state) funds.

second: separate specialising insurance companies must not concern themselves with insurance activities, for the formation of such insurance companies economic calculations could be accounted on; viz: capacity, low cost of insurance service.

third: Closely connected to insurance activities are other methods which lead to the regulation of the development process of the National Economy, for instance, institutions aiming at the elimination of disturbances occurring. The choice in a concrete instance of this, or other regulating methods, is again exclusively a true economic problem which, consequently, depends on the costs and the capacity of the regulator.

Possible use of the methods of cybernetic economy in the insurance field

The cybernetic insurance theory that is held to consider insurance as a regulator for the development process of National Economy threatened by prejudicial events, tolerates the application of the analysis of economic results of the insurance activity as well as universal methods of automatic regulation theory. That is cybernetica, which incidentally is already practised favourably in other branches of technical, economic and social activities.

When practising the methods of automatic regulation, the system of National Economy can be imagined as a collection of blocks (elements) which are connected to each other by antipodean effect of the causal chain. Each block of the system e.g.: individual enterprises, land- or Property-economy, has a special task within the framework of the social and economical systems. On each block other blocks and environments are working in a particular way at the time when the respective block is in position. This environment is to be considered as one or various blocks in the system. Each block is influencing in one way or another some or all of the other blocks. Within the block systems, through transformation, the 
block's "Input position" is taking place on an "Output Position". Such a transformation in a production enterprise can signify for instance, the transformation of the received production means on one or more products. A rule, or a collection of rules, through which the transformation "Input Position" on "Output Position" is effected, bears the name of a transformation-function of the block. If the position "Block-Input" with the assistance of an ordered collection of true cyphers is $x_{1}, x_{2}, \ldots \ldots \ldots x_{m}$, i.e. with the aid of vector $x=\left(x_{1}, x_{2}, \ldots \ldots \ldots x_{m}\right)$, the transformation function of the block can be indicated with the symbol $y=T x$, where $y$ signifies the ordered collection of the entry-values, thus vector $y=\left(y_{1}, y_{2}, \ldots \ldots \ldots y_{n}\right)$.

In the transformation-function $y=T x$ without the independent variable, (i.e. the intruded elements) of the block $x_{1}, x_{2}, \ldots \ldots \ldots$, $x_{m}$ and of the dependent variable $y_{1}, y_{2} \ldots \ldots, y_{n}$ the parameters $a_{1}, a_{2}, \ldots \ldots, a_{s}$, appear, which determine the inner characteristics, thus proper distinguishing marks, and the parameters $b_{1}, b_{2}, \ldots \ldots \ldots, b_{r}$, which determine the position of the environment, thus the ratio, wherein the transformation of the block is effected. In this respect it is assumed that the parameters which characterize the inner qualities of the block and its environment are known and constant--at least for a certain short period of time. If the systemblocks are production enterprises, the independent variable $x_{1}, x_{2}, \ldots \ldots, x_{m}$, will indicate the size of the individual production factors and the dependent variable $y_{1}, y_{2}, \ldots \ldots \ldots, y_{n}$, the size of individual products. The parameters $a_{1}, a_{2}, \ldots \ldots \ldots$, indicate the technical organisation of the enterprise in question (amongst others, technical coefficients of the production, power of production, the stocks of raw material) and the parameters $b_{1}, b_{2}, \ldots \ldots, b_{r}, a_{s}$, indicate such factors as temperature, weather elements, prices of raw materials, selling prices of the products, as well as other circumstances of the environment. The independent variables together with the parameters appearing in the transformation-function, of which some or all, can equally be a variable of events with the known grouping of the probability. Equally possible is the hypothesis that some independent variables of the parameters can bear the character of strategie variables. In proportion to the character of the variables and of the parameters 
of the block we have to deal with the transformation of an estimated or non-estimated risk. When judging the deviations caused in the block, we have to apply in the first case the method of probability calculation and of mathematical statistics, and in the second case, methods of the theory of strategic games.

Each block $S$ or a group of similar block systems is provided with the regulator $R$, which is connected to the rear of the regulated block. This, as is known, is the control method of the respective system into which enter the results of preceding effects. Within the technique both blocks $S$ and $R$ are produced by the human element. The regulated block $S$ within the national economy is the objective social-economic process through which exit appear increases or decreases in the standard of living, increased consumption, population growth etc. Regulators, on the other hand, are certain institutions, which are within a socialist sphere carried out and guided by the State, and equally in other social systems by groups or individual persons.

Regulator $R$ protects the appointed end of the process that has taken place in block $S$. The basic task of the regulator is in fact to protect the stability of the regulated block. This above all is shown by equalisation of the deviations of block $S$ from the scheduled purpose whether it be with the assistance of the cypher or the vector $z$, or the time-function $z(t)$ (programme-guiding).

In cases of deviation arising in the regulated block $S$ due to occurrences, it is fundamentally as a regulator just one economical institution, called insurance. From this it is apparent that the size of the insurance funds, natural or financial resources, can be determined by similar methods as is applied in the theory of automatic regulation to appoint the power of the regulator. Proof of this kind is at the moment being taken by Polish actuaries in applying some methods of programming valued and non-valued proportions of risks. In particular the methods of dynamic programming which serve for the intended amount of stocks (reserves), can find a more ample application in uncertain conditions.

The starting point here is the fundamental formula of the regulation theory $y=\frac{S}{\mathrm{I}-S R} x$, in which $S$ and $R$ are the linear operators of the line transformation. This accordingly is effected in 
the $S$ regulated block and the regulator $R$. If, on account of accidental disturbances within the system, the "Output position" of the block under consideration $y$ is different to the adjusted volume, that is of the norm $z$, there will appear static or dynamic deviations of the block $y-z$. These deviations can be taken off in two different ways:

I. through improvement of the adjusted volume $x$, i.e. of the intruded elements of the regulated block $S$, or

2. through reconstruction of the regulator, or alternatively by inserting an additional regulator into the system.

In order to reach the equilibrium of the regulated block, the fundamental formula in the regulation theory and in accordance herewith the resulting adjustment of the block reaction, allows for either the determination of the intruded block elements, or the power of the regulator. In the insurance field, fundamentally, we have to introduce the second means of regulation which is dependent on the formation of a substantial insurance fund.

The general theory of regulation and its accompanying theory of reliability, which is based on the structure of a reliable system, composed of non-reliable blocks (elements), confirms also the fact, that the construction of joint regulators is a better achievement for the whole system than the construction of separate regulators for each special blocksystem. This conclusion expressed in insurance language simply says that the insurance fund, necessary for the regulation of the economic processes, is decreasing proportionately with the growth of insured objects.

\section{Practical conclusions resulting from the cybernetic insurance theory}

The cybernetic insurance theory allows-as it appears-a more universal theoretical image of the economic role of insurance, and opens new ways for the application of contemporary mathematicstatistical methods in insurance calculation.

Fom this theory also results several further conclusions of more practical nature, viz.:

I. The regulation system of social-economic processes based on the compensation of unfavourable effects caused by disturbances, is closely connected to other regulation methods. Regulation in the widest sense of the word relies not only on the equalization of the 
caused deviations in the course of this process to achieve the ultimate and desired end. Regulation can also-wholly or partially -be accomplished by eliminating disturbances, i.e. by not allowing such disturbances to arise in the system under consideration. It is also possible to guide the compensation method for disturbances, which differs from the elimination method of disturbances in such a way that it is trying to weaken the already occurred disturbances, or to compensate them fully.

From the economic account results in the first instance, the appiication within a larger or smaller field of these three methods, which are battling for protection against the effects of unfavourable events, in conformity with the standard of the total National Economy. It would seem to be most useful to apply methods which could indeed eliminate occurring disturbances, i.e. loss prevention methods, or methods that compensate disturbances, thus the socalled extinguishing methods. In practice, however, it appears that in cases of events the effect of both preventing and extinguishing methods is limited. Besides, very little effect is frequently gained from the application of these methods seen from the viewpoint of the economic account. This means, for example that the diminishing of the loss caused by an event can turn out to be much less costly than that for loss prevention. In such cases it is necessary to apply insurance methods of loss-compensation.

In many cases it is useful-seen from the view-point of the complete National Economy-to apply unknown regulation methods with ultimate directions and extensions of the individual regulation methods. This can be determined with the aid of linear programming or with other methods of scientific operation (industrial research).

2. Relying on the cybernetic insurance theory, one could, looking from an economic view-point, also determine the most powerful organisation of the insurance activity.

By regulation capacity - this being the characteristic of insurance work-is to be understood:

a) quick results, i.e. the space of time in which the liquidation of arising disturbances is executed within the stable block,

b) precision (correct methods) of the effective working-measured with the difference which notwithstanding the application of the 
regulation arises between the "outgoing" level of block $y$ in the position of equilibrium and the appointed extent $z$, and

c) reliability of regulation, determined by the probability $p=\mathbf{I}-q$ where $q$ indicates the probability that the regulator fails in the given time.

The economic cybernetic allows for estimating the extent of these elements, therefore, to determine the way in which conditions at the regulator have to proceed in order to fulfil its working efficiency to the ultimate possible end.

From this analysis results the optimal structure of the organisation of the Insurance Theory--thus determining whether responsibility is falling to the State or to one or more other specialised insurance institutions.

3. Lastly - but by no means least - advantages of the cybernetic redaction of the insurance theory rely on the possibility of application of the simulation theory in order to pursue the foreseen course of events, and estimation of losses in the group of insured objects. There is also the possibility of an experimental examination into alterations occurring in the group of objects similar to that as is executed in the groups of persons (generations) in life- and accident insurance. Here can be applied methods of mathematical demography and experiments with the aid of electronic computers inclining to the method "Monte Carlo".

The theory of simulation allows one to determine whether the power of the regulator, which is Insurance, will be sufficiently strong to restore the balance of the whole National Economy which is disturbed by certain events and its constructions (economy units).

Interpreted into common insurance-language, this thought leads to the fact that the applied methods of simulation in the cybernetic insurance theory determine the extent of the insurance fund, and its formation, in a period sufficient for a quick and full coverage of losses.

\section{Summary}

The present referendum is exclusively aimed at the purpose of highlighting other possibilities of a theoretical approach of judgment to the social-economic rôle of insurance, in particular with regard to the socialist economy. The cybernetic insurance theory differs 
fundamentally from traditional theories, if only for not claiming a "Risk Community" necessity, i.e. a group of persons or special economy units, the capital of which is threatened by a common danger.

Nevertheless, the development of the economic process needs, even within the sphere of socialist economy, the application of regulating institutions. One of such economic institutions, is insurance.

In this referendum conclusions have also been drawn on the practical use of cybernetic insurance theory in insurance calculations. To proceed working out this thesis in greater detail would be beyond the scope of this referendum. 